Appl. Set-Valued Anal. Optim. 2 (2020), No. 1, pp. 49-61

Available online at http://asvao.biemdas.com

https://doi.org/10.23952/asvao.2.2020.1.04

\title{
BOUNDED PERTURBATION RESILIENCE OF GENERALIZED VISCOSITY ITERATIVE ALGORITHMS FOR SPLIT VARIATIONAL INCLUSION PROBLEMS
}

\author{
PEICHAO DUAN ${ }^{1,2 *}$, XUBANG ZHENG $^{2}$ \\ ${ }^{1}$ College of Science, Civil Aviation University of China, Tianjin 300300, China \\ ${ }^{2}$ Tianjin Key Lab for Advanced Signal Processing, Civil Aviation University of China, Tianjin 300300, China
}

\begin{abstract}
In this paper, we propose a generalized viscosity approximation method combing a sequence of contractive mappings to solve a split variational inclusion problem. The bounded perturbation resilience of the method is investigated in Hilbert spaces. Under mild conditions, we prove that our algorithms strongly converge to a solution of the split variational inclusion problem, which is also the unique solution of some variational inequality problem. Furthermore, we show the convergence and effectiveness of the algorithms via two numerical examples. Our results extend and improve the related results in the literature.
\end{abstract}

Keywords. Split variational inclusion problem; Maximal monotone operator; Contractive mapping; Viscosity iteration.

\section{INTRODUCTION}

Let $H_{1}$ and $H_{2}$ be two real Hilbert spaces with inner product $\langle$,$\rangle and induced norm \|\cdot\|$. In this paper, we deal with the split variational inclusion problem (SVIP, for short) of the following type: Find a point $x^{*} \in H_{1}$ such that

$$
0 \in B_{1}\left(x^{*}\right)
$$

and $y^{*}=A x^{*} \in H_{2}$ solves

$$
0 \in B_{2}\left(y^{*}\right),
$$

where $A: H_{1} \rightarrow H_{2}$ is a bounded linear operator, $B_{1}: H_{1} \rightarrow 2^{H_{1}}$ and $B_{2}: H_{2} \rightarrow 2^{H_{2}}$ are maximal monotone mappings. We denote the solution set of SVIP (1.1)-(1.2) by

$$
S=\left\{x^{*} \in H_{1}: 0 \in B_{1}\left(x^{*}\right) \text { and } A x^{*} \in H_{2}: 0 \in B_{2}\left(A x^{*}\right)\right\} .
$$

The SVIP has been applied to solving many real life problems, such as, modelling intensitymodulated radiation therapy treatment planning [7, 8], and modelling of inverse problems arising from phase retrieval [4]. Recently, a lot of projection algorithms were proposed to solve the SVIP (1.1)-(1.2) in infinite-dimensional spaces; see, e.g., [2, 5, 6, 11, 12, 13, 23, 25] and the references therein.

In 2012, Byrne et al. [5] gave the following two algorithms for the split variational inclusion problem: For any $x_{0} \in H_{1},\left\{x_{n}\right\}$ is a sequence defined as follows

$$
x_{n+1}=J_{\lambda}^{B_{1}}\left(x_{n}-\tau A^{*}\left(I-J_{\lambda}^{B_{2}}\right) A x_{n}\right)
$$

\footnotetext{
*Corresponding author.

E-mail addresses: pcduancauc@126.com (P. Duan), xbzheng224@163.com (X. Zheng).

Received August 23, 2019; Accepted December 4, 2019.
} 
and

$$
x_{n+1}=\alpha_{n} u+\left(1-\alpha_{n}\right) J_{\lambda}^{B_{1}}\left(x_{n}-\tau A^{*}\left(I-J_{\lambda}^{B_{2}}\right) A x_{n}\right) .
$$

Under appropriate conditions, they obtained weak and strong convergence theorems, respectively, in the framework of Hilbert spaces.

The following lemma is very useful for constructing out iterative algorithms.

Lemma 1.1. [26] Let $H_{1}$ and $H_{2}$ be Hilbert spaces. Let $B: H_{1} \rightarrow 2^{H_{1}}$ be a maximal monotone mapping and let $J_{\lambda}^{B}=(I+\lambda B)^{-1}$ be the resolvent of $B$ for $\lambda>0$. Let $T: H_{2} \rightarrow H_{2}$ be a nonexpansive mapping and let $A: H_{1} \rightarrow H_{2}$ be a bounded linear operator. Suppose that $B^{-1} 0 \cap$ $A^{-1} \mathrm{Fix}(T) \neq \emptyset$. Then the following equality holds:

$$
\operatorname{Fix}\left(J_{\lambda}^{B}\left(I-\tau A^{*}(I-T) A\right)\right)=B^{-1} 0 \cap A^{-1} F i x(T),
$$

where $\lambda$ and $\tau$ are two real numbers.

In 2014, Kazmi and Rizvi [21] proposed the following iteration algorithm

$$
\left\{\begin{array}{l}
y_{n}=J_{\lambda}^{B_{1}}\left(x_{n}-\tau A^{*}\left(I-J_{\lambda}^{B_{2}}\right) A x_{n}\right), \\
x_{n+1}=\alpha_{n} h\left(x_{n}\right)+\left(1-\alpha_{n}\right) T y_{n} .
\end{array}\right.
$$

They proved that the sequence $\left\{x_{n}\right\}$ generated by the above algorithm converges strongly a common element both in the fixed-point set of nonexpansive mapping $T$ and the solution set of SVIP (1.1)-(1.2).

On the other hand, the bounded perturbation resilience and superiorization of iterative methods are extensively studied in many references, for example, [3, 9, 16, 18, 20, 29]. The problem has received much attention due to its applications in convex feasibility problems [10], image reconstruction [15] and inverse problems of radiation therapy [14], and so on.

An algorithm operator $\mathbf{P}$ is said to be bounded perturbation resilient [9] if the iteration $x_{n+1}=$ $\mathbf{P} x_{n}$ is replaced by $x_{n+1}=\mathbf{P}\left(x_{n}+\beta_{n} v_{n}\right)$, where $\beta_{n} \geq 0$ for all $n \geq 0$ and $v_{n} \in H$ with

$$
\sum_{n=0}^{\infty} \beta_{n}<\infty \text { and }\left\|v_{n}\right\| \leq M \text {. }
$$

is still convergent.

Consider the following minimization problem:

$$
\min _{x \in C} f(x),
$$

where $f$ is a continuous differentiable, convex function. $\mathrm{Xu}$ [29] proposed the superiorization techniques for the relaxed projected scaled gradient method. Xu's algorithm is as follows

$$
x_{n+1}=\left(1-\tau_{n}\right) x_{n}+\tau_{n} P_{C}\left(x_{n}-\gamma_{n} D\left(x_{n}\right) \nabla f\left(x_{n}\right)+e\left(x_{n}\right)\right), n \geq 0,
$$

where $\tau_{n}$ is a sequence in $[0,1]$, and $D\left(x_{n}\right)$ is a diagonal scaling matrix. He established weak convergence of the above algorithm in [29] under appropriate conditions imposed on $\left\{\tau_{n}\right\}$, and $\left\{\gamma_{n}\right\}$.

Very recently, Guo and Cui [18] introduced a modified proximal gradient algorithm with bounded perturbations. Indeed, their iterative sequence $\left\{x_{n}\right\}$ is generated as follows

$$
x_{n+1}=t_{n} h\left(x_{n}\right)+\left(1-t_{n}\right) \operatorname{prox}_{\gamma_{n}}\left(x_{n}-\gamma_{n} \nabla f\left(x_{n}\right)+e\left(x_{n}\right)\right), \quad n \geq 0 .
$$

Under suitable conditions, they obtained solution theorems in Hilbert spaces. 
In this paper, based on the works [9, 26, 29], we propose a generalized viscosity approximation method for solving SVIP (1.1)-(1.2). We show that our iterative algorithm is bounded perturbation resilience and show the convergence point of the iterative algorithm which is also the unique solution of some variational inequality problem. Finally, two numerical examples are given to demonstrate the effectiveness of our iterative algorithms:

$$
x_{n+1}=\alpha_{n} h_{n}\left(x_{n}\right)+\left(1-\alpha_{n}\right) J_{\lambda}^{B_{1}}\left(x_{n}-\tau_{n} A^{*}\left(I-J_{\lambda}^{B_{2}}\right) A x_{n}+e\left(x_{n}\right)\right),
$$

where $\left\{h_{n}\right\}$ is a sequence of contractive mappings. We will show that the superiorization of (1.8) yields a sequence $\left\{x_{n}\right\}$ generated by the following iterative process:

$$
\left\{\begin{array}{l}
y_{n}=x_{n}+\beta_{n} v_{n}, \\
x_{n+1}=\alpha_{n} h_{n}\left(y_{n}\right)+\left(1-\alpha_{n}\right) J_{\lambda}^{B_{1}}\left(\left(I-\tau_{n} A^{*}\left(I-J_{\lambda}^{B_{2}}\right) A\left(y_{n}\right)+e\left(y_{n}\right)\right),\right.
\end{array}\right.
$$

In addition, we also discuss the convergence of the generalized viscosity algorithm and prove that it is bounded perturbation resilient.

\section{Preliminaries}

We adopt the following notations:

(1) $x_{n} \rightarrow x$ indicates that $\left\{x_{n}\right\}$ is strongly convergent to $x ; x_{n} \rightarrow x$ indicates that $\left\{x_{n}\right\}$ is weakly convergent to $x$.

(2) Fix $(T)$ denotes the set of fixed points of $T$, that is, $\operatorname{Fix}(T)=\{x \in H: T x=x\}$.

(3) $\omega_{w}\left(x_{n}\right):=\left\{x: \exists x_{n_{j}} \rightarrow x\right\}$ denotes the weak $\omega$-limit set of $\left\{x_{n}\right\}$.

We list the following lemmas, definitions and propositions, which are needed in the proof of main results.

Lemma 2.1. Let $H$ be a real Hilbert space. There holds the following inequality:

$$
\|x-y\|^{2} \leq\|x\|^{2}+2\langle x+y, y\rangle, \forall x, y \in H .
$$

Definition 2.1. A mapping $F: H \rightarrow H$ is said to be

(i) Lipschizian if there exists a positive constant $L$ such that

$$
\|F x-F y\| \leq L\|x-y\|, \quad \forall x, y \in H .
$$

In particular, if $L=1$, we say that $F$ is nonexpansive, that is,

$$
\|F x-F y\| \leq\|x-y\|, \forall x, y \in H .
$$

If $L \in[0,1)$, we say that $F$ is contractive.

(ii) $\alpha$-averaged mapping ( $\alpha$-av, for short) if

$$
F=(1-\alpha) I+\alpha T
$$

where $\alpha \in(0,1)$ and $T: H \rightarrow H$ is nonexpansive.

Definition 2.2. A mapping $B: H \rightarrow H$ is said to be

(i) monotone if

$$
\langle B x-B y, x-y\rangle \geq 0, \forall x, y \in H .
$$

(ii) $\eta$-strongly monotone if there exists a positive constant $\eta$ such that

$$
\langle B x-B y, x-y\rangle \geq \eta\|x-y\|^{2}, \quad \forall x, y \in H .
$$


(iii) $\alpha$-inverse strongly monotone ( $\alpha$-ism, for short) if there exists a positive constant $\alpha$ such that

$$
\langle B x-B y, x-y\rangle \geq \alpha\|B x-B y\|^{2}, \forall x, y \in H .
$$

In particular, if $\alpha=1$, we say that $B$ is firmly nonexpansive, namely,

$$
\langle B x-B y, x-y\rangle \geq\|B x-B y\|^{2}, \forall x, y \in H .
$$

Definition 2.3. Let a mapping $B: H \rightarrow H$ be monotone. Then $B$ is maximal monotone if there exists no monotone operator $A: H \rightarrow 2^{H}$ such that graA properly contains graB, i.e., for every $(x, u) \in H \times H$,

$$
(x, u) \in \operatorname{graB} \Leftrightarrow \forall(y, v) \in \operatorname{graB},\langle x-y, u-v\rangle \geq 0 .
$$

Lemma 2.2. [28] Let $h: H \rightarrow H$ be a $\rho$-contraction with $\rho \in(0,1)$ and $T: H \rightarrow H$ be a nonexpansive mapping. Then

(i) $I-h$ is $(1-\rho)$-strongly monotone:

$$
\langle(I-h) x-(I-h) y, x-y\rangle \geq(1-\rho)\|x-y\|^{2}, \quad \forall x, y \in H .
$$

(ii) $I-T$ is monotone:

$$
\langle(I-T) x-(I-T) y, x-y\rangle \geq 0, \quad \forall x, y \in H .
$$

Proposition 2.1. [27]

(i) If $T_{1}, T_{2}, \cdots, T_{n}$ are averaged mappings, we have that $T_{n} T_{n-1} \cdots T_{1}$ is averaged. In particular, if $T_{i}$ is $\alpha_{i}-a v, i=1,2$, where $\alpha_{i} \in(0,1)$, then $T_{2} T_{1}$ is $\left(\alpha_{2}+\alpha_{1}-\alpha_{2} \alpha_{1}\right)-a v$.

(ii) If the mappings $\left\{T_{i}\right\}_{i=1}^{N}$ are averaged and have a common fixed point, then,

$$
\bigcap_{i=1}^{N} F i x\left(T_{i}\right)=F i x\left(T_{1} \ldots T_{N}\right) \text {. }
$$

(iii) A mapping $T$ is nonexpansive if and only if $I-T$ is $\frac{1}{2}-i s m$.

(iv) If $T$ is $v$-ism, then, for $\tau>0$, $\tau T$ is $\frac{v}{\tau}-i s m$.

(v) $T$ is averaged if and only if $I-T$ is $v$-ism for some $v>\frac{1}{2}$. Indeed, for $0<\alpha<1, T$ is $\alpha$-averaged if and only if $I-T$ is $\frac{1}{2 \alpha}-i s m$.

Lemma 2.3. [17] Let $H$ be a real Hilbert space, and let $T: H \rightarrow H$ be a nonexpansive mapping with Fix $(T) \neq \emptyset$. If $\left\{x_{n}\right\}$ is a sequence in $H$ weakly converging to $x$ and if $\left\{(I-T) x_{n}\right\}$ converges strongly to $y$, then $(I-T) x=y$; in particulary, if $y=0$, then $x \in F i x(T)$.

Lemma 2.4. (see $[19,30]$ ) Assume that $\left\{s_{n}\right\}$ is a sequence of nonnegative real numbers such that

$$
\begin{gathered}
s_{n+1} \leq\left(1-\gamma_{n}\right) s_{n}+\gamma_{n} \delta_{n}, n \geq 0, \\
s_{n+1} \leq s_{n}-\eta_{n}+\varphi_{n}, \quad n \geq 0,
\end{gathered}
$$

where $\left\{\gamma_{n}\right\}$ is a sequence in $(0,1),\left(\eta_{n}\right)$ is a sequence of nonnegative real numbers and $\left(\delta_{n}\right)$ and $\left(\varphi_{n}\right)$ are two sequences in $\mathbb{R}$ such that

(i) $\sum_{n=0}^{\infty} \gamma_{n}=\infty$,

(ii) $\lim _{n \rightarrow \infty} \varphi_{n}=0$,

(iii) $\lim _{k \rightarrow \infty} \eta_{n_{k}}=0$ implies $\limsup _{k \rightarrow \infty} \delta_{n_{k}} \leq 0$ for any subsequence $\left(n_{k}\right) \subset(n)$.

Then $\lim _{n \rightarrow \infty} s_{n}=0$. 


\section{MAIN RESUlTS}

In 2000, Moudafi [22] proposed the viscosity approximation method for a nonexpansive mapping $T$ with a nonempty fixed-point set $\operatorname{Fix}(T)$ in a Hilbert space $H$ :

$$
x_{n+1}=\alpha_{n} h\left(x_{n}\right)+\left(1-\alpha_{n}\right) T x_{n},
$$

where $h: H \rightarrow H$ is $\rho$-contractive mapping and $\left\{\alpha_{n}\right\} \subset(0,1)$ is a real sequence, and proved that the sequence $\left\{x_{n}\right\}$ converges strongly to a fixed point of the mapping $T$ if the sequence $\left\{\alpha_{n}\right\}$ satisfies appropriate conditions. It was also proved that the fixed point also uniquely solves the following variational inequality:

$$
\left\langle(I-h) x^{*}, \tilde{x}-x^{*}\right\rangle \geq 0, \forall \tilde{x} \in F i x(T) .
$$

Recently, the viscosity approximation method have been extended and studied in the framework of Banach spaces; see [24, 28] and the references therein.

Re-writing algorithm (1.8), one has

$$
\begin{aligned}
x_{n+1} & =\alpha_{n} h_{n}\left(x_{n}\right)+\left(1-\alpha_{n}\right) J_{\lambda}^{B_{1}}\left(x_{n}-\tau_{n} A^{*}\left(I-J_{\lambda}^{B_{2}}\right) A x_{n}+e\left(x_{n}\right)\right) \\
& =\alpha_{n} h_{n}\left(x_{n}\right)+\left(1-\alpha_{n}\right)\left(J_{\lambda}^{B_{1}}\left(x_{n}-\tau_{n} A^{*}\left(I-J_{\lambda}^{B_{2}}\right) A x_{n}\right)+\tilde{e}_{n}\right) .
\end{aligned}
$$

This algorithm is a outer perturbed version. Since $J_{\lambda_{n}}^{B_{1}}$ is nonexpansive, we have

$$
\begin{aligned}
\left\|\tilde{e}_{n}\right\| & =\left\|J_{\lambda}^{B_{1}}\left(x_{n}-\tau_{n} A^{*}\left(I-J_{\lambda}^{B_{2}}\right) A x_{n}+e\left(x_{n}\right)\right)-J_{\lambda}^{B_{1}}\left(x_{n}-\tau_{n} A^{*}\left(I-J_{\lambda}^{B_{2}}\right) A x_{n}\right)\right\| \\
& \leq\left\|e\left(x_{n}\right)\right\| .
\end{aligned}
$$

Next, we use $S$ to denote the solution set of the SVIP (1.1)-(1.2) and use the generalized viscosity iterative method to find solutions of the SVIP (1.1)-(1.2).

Theorem 3.1. Let $H_{1}, H_{2}$ be two real Hilbert spaces and $A$ be a bounded linear operator with $L=\left\|A^{*} A\right\|$, where $A^{*}$ is the adjoint of $A$. Suppose that $B_{1}: H_{1} \rightarrow 2^{H_{1}}$ and $B_{2}: H_{2} \rightarrow 2^{H_{2}}$ are maximal monotone operators and $S \neq \emptyset$. Let $h_{n}$ be a $\rho_{n}$-contractive self-mapping of $H_{1}$ with $0 \leq \rho_{l}=\liminf _{n \rightarrow \infty} \rho_{n} \leq \limsup _{n \rightarrow \infty} \rho_{n}=\rho_{u}<1$. Suppose that the contractive mapping sequence $\left\{h_{n}(x)\right\}$ is uniformly convergent for any $x \in E$, where $E$ is any bounded subset of $H_{1}$. Given $x_{0} \in H_{1}$, define the sequence $\left\{x_{n}\right\}$ by

$$
x_{n+1}=\alpha_{n} h_{n}\left(x_{n}\right)+\left(1-\alpha_{n}\right) J_{\lambda}^{B_{1}}\left(x_{n}-\tau_{n} A^{*}\left(I-J_{\lambda}^{B_{2}}\right) A x_{n}+e\left(x_{n}\right)\right),
$$

where $\tau_{n} \in\left(0, \frac{2}{L}\right)$. Assume the following conditions hold:

(i) $\lim _{n \rightarrow \infty} \alpha_{n}=0$;

(ii) $\sum_{n=0}^{\infty} \alpha_{n}=\infty$;

(iii) $0<\liminf _{n \rightarrow \infty} \tau_{n} \leq \limsup _{n \rightarrow \infty} \tau_{n}<\frac{2}{L}$;

(iv) $\sum_{n=0}^{\infty}\left\|e\left(x_{n}\right)\right\|<\infty$.

Then $\left\{x_{n}\right\}$ converges strongly to $x^{*}$, where $x^{*}$ is a solution of the SVIP (1.1)-(1.2), which is also the unique solution of variational inequality problem (3.1).

Proof. Setting

$$
V_{\tau_{n}}:=J_{\lambda}^{B_{1}}\left(I-\tau_{n} A^{*}\left(I-J_{\lambda}^{B_{2}}\right) A\right),
$$

we have that $V_{\tau_{n}}$ is $\frac{2+\tau_{n} L}{4}-a v$. Note that $I-\tau_{n} A^{*}\left(I-J_{\lambda}^{B_{2}}\right) A$ is $\frac{\tau_{n} L}{2}-a v$ as $0<\tau_{n}<2 / L$ and $J_{\lambda}^{B_{1}}$ is $\frac{1}{2}-$ av [1]. It follows from Proposition 2.1 that the composite $V_{\tau_{n}}$ is $\frac{2+\tau_{n} L}{4}-a v$. 
Next, we split the proof into several steps.

Step 1. Show that $\left\{x_{n}\right\}$ is bounded.

For any $z \in S$, we get

$$
\begin{aligned}
\left\|x_{n+1}-z\right\| & =\left\|\alpha_{n} h_{n}\left(x_{n}\right)+\left(1-\alpha_{n}\right)\left(V_{\tau_{n}} x_{n}+\tilde{e}_{n}\right)-z\right\| \\
& =\left\|\alpha_{n}\left(h_{n}\left(x_{n}\right)-z\right)+\left(1-\alpha_{n}\right)\left(V_{\tau_{n}} x_{n}-z\right)+\left(1-\alpha_{n}\right) \tilde{e}_{n}\right\| \\
& \leq \alpha_{n}\left\|h_{n}\left(x_{n}\right)-h_{n}(z)\right\|+\alpha_{n}\left\|h_{n}(z)-z\right\|+\left(1-\alpha_{n}\right)\left\|V_{\tau_{n}} x_{n}-z\right\|+\left\|\tilde{e}_{n}\right\| \\
& \leq \alpha_{n} \rho_{n}\left\|x_{n}-z\right\|+\alpha_{n}\left\|h_{n}(z)-z\right\|+\left(1-\alpha_{n}\right)\left\|x_{n}-z\right\|+\left\|\tilde{e}_{n}\right\| \\
& =\left(1-\alpha_{n}\left(1-\rho_{n}\right)\right)\left\|x_{n}-z\right\|+\alpha_{n}\left(1-\rho_{n}\right) \frac{\left\|h_{n}(z)-z\right\|+\left\|\tilde{e}_{n}\right\| / \alpha_{n}}{1-\rho_{n}} .
\end{aligned}
$$

Since $\left\{h_{n}\right\}$ is uniformly convergent on bounded sets, it is easy to get that $\left\{h_{n}(z)\right\}$ is bounded. From the conditions (ii), (iv) and $\alpha_{n}>0$, we find that $\left\{\left\|\tilde{e}_{n}\right\| / \alpha_{n}\right\}$ is also bounded. Thus, there exists some $M_{1}>0$ such that $\sup \left\{\left\|h_{n}(z)-z\right\|+\left\|\tilde{e}_{n}\right\| / \alpha_{n}\right\} \leq M_{1}$ for all $n \geq 0$. It follows that

$$
\left\|x_{n}-z\right\| \leq \max \left\{\left\|x_{0}-z\right\|, \frac{M_{1}}{1-\rho_{u}}\right\}
$$

which implies that sequence $\left\{x_{n}\right\}$ is bounded, so are $\left\{h_{n}\left(x_{n}\right)\right\},\left\{V_{\tau_{n}} x_{n}\right\}$ and $\left\{A^{*}\left(I-J_{\lambda}^{B_{2}}\right) A x_{n}\right\}$. Step 2. Show that, for any sequence $\left(n_{k}\right) \subset(n)$, if $\eta_{n_{k}} \rightarrow 0$, then $\lim _{k \rightarrow \infty}\left\|x_{n_{k}}-V_{\tau_{n_{k}}} x_{n_{k}}\right\|=0$.

Fixing $z \in S$, we have

$$
\begin{aligned}
& \left\|x_{n+1}-z\right\|^{2} \\
& \leq\left\|\alpha_{n} h_{n}\left(x_{n}\right)+\left(1-\alpha_{n}\right) V_{\tau_{n}} x_{n}-z\right\|^{2}+2\left\langle\alpha_{n} h_{n}\left(x_{n}\right)+\left(1-\alpha_{n}\right) V_{\tau_{n}} x_{n}-z,\left(1-\alpha_{n}\right) \tilde{e}_{n}\right\rangle+\left\|\tilde{e}_{n}\right\|^{2} \\
& \leq \alpha_{n}^{2}\left\|h_{n}\left(x_{n}\right)-z\right\|^{2}+\left(1-\alpha_{n}\right)^{2}\left\|V_{\tau_{n}} x_{n}-z\right\|^{2}+2 \alpha_{n}\left(1-\alpha_{n}\right)\left\langle h_{n}\left(x_{n}\right)-z, V_{\tau_{n}} x_{n}-z\right\rangle \\
& +\left(2 \alpha_{n}\left\|h_{n}\left(x_{n}\right)-z\right\|+2\left(1-\alpha_{n}\right)\left\|x_{n}-z\right\|+\left\|\tilde{e}_{n}\right\|\right)\left\|\tilde{e}_{n}\right\| \\
& \leq 2 \alpha_{n}^{2}\left(\left\|h_{n}\left(x_{n}\right)-h_{n}(z)\right\|^{2}+\left\|h_{n}(z)-z\right\|^{2}\right)+\left(1-\alpha_{n}\right)^{2}\left\|V_{\tau_{n}} x_{n}-z\right\|^{2} \\
& +2 \alpha_{n}\left(1-\alpha_{n}\right)\left\langle h_{n}\left(x_{n}\right)-z, V_{\tau_{n}} x_{n}-z\right\rangle+M_{2}\left\|\tilde{e}_{n}\right\| \\
& \leq 2 \alpha_{n}^{2}\left(\left\|h_{n}\left(x_{n}\right)-h_{n}(z)\right\|^{2}+\left\|h_{n}(z)-z\right\|^{2}\right)+\left(1-\alpha_{n}\right)^{2}\left\|V_{\tau_{n}} x_{n}-z\right\|^{2} \\
& +2 \alpha_{n}\left(1-\alpha_{n}\right)\left(\left\|h_{n}\left(x_{n}\right)-h_{n}(z)\right\|\left\|x_{n}-z\right\|+\left\langle h_{n}(z)-z, V_{\tau_{n}} x_{n}-z\right\rangle\right)+M_{2}\left\|\tilde{e}_{n}\right\| \\
& \leq\left(1-\alpha_{n}\left(2-\alpha_{n}\left(1+2 \rho_{n}^{2}\right)-2\left(1-\alpha_{n}\right) \rho_{n}\right)\right)\left\|x_{n}-z\right\|^{2}+2 \alpha_{n}\left(1-\alpha_{n}\right)\left\langle h_{n}(z)-z, V_{\tau_{n}} x_{n}-z\right\rangle \\
& +2 \alpha_{n}^{2}\left\|h_{n}(z)-z\right\|^{2}+M_{2}\left\|\tilde{e}_{n}\right\| \\
& \leq\left(1-\alpha_{n}\left(2-\alpha_{n}\left(1+2 \rho_{u}^{2}\right)-2\left(1-\alpha_{n}\right) \rho_{u}\right)\right)\left\|x_{n}-z\right\|^{2}+2 \alpha_{n}\left(1-\alpha_{n}\right)\left\langle h_{n}(z)-z, V_{\tau_{n}} x_{n}-z\right\rangle \\
& +2 \alpha_{n}^{2}\left\|h_{n}(z)-z\right\|^{2}+M_{2}\left\|\tilde{e}_{n}\right\|
\end{aligned}
$$

where

$$
M_{2}=\sup \left\{2 \alpha_{n}\left\|h_{n}\left(x_{n}\right)-z\right\|+2\left(1-\alpha_{n}\right)\left\|x_{n}-z\right\|+\left\|\tilde{e}_{n}\right\|\right\} .
$$

Since $V_{\tau_{n}}$ is $\frac{2+\tau_{n} L}{4}-a v$, we can rewrite

$$
V_{\tau_{n}}=J_{\lambda}^{B_{1}}\left(I-\tau_{n} A^{*}\left(I-J_{\lambda}^{B_{2}}\right) A\right)=\left(1-w_{n}\right) I+w_{n} T_{n},
$$


where $w_{n}=\frac{2+\tau_{n} L}{4}, T_{n}$ is a nonexpansive mapping. From condition (iii), we have

$$
\frac{1}{2}<\liminf _{n \rightarrow \infty} w_{n} \leq \limsup _{n \rightarrow \infty} w_{n}<1 .
$$

Thus, it follows from (3.2) and (3.6) that

$$
\begin{aligned}
\left\|x_{n+1}-z\right\|^{2}= & \left\|\alpha_{n} h_{n}\left(x_{n}\right)+\left(1-\alpha_{n}\right)\left(V_{\tau_{n}} x_{n}+\tilde{e}_{n}\right)-z\right\|^{2} \\
\leq & \left\|\alpha_{n} h_{n}\left(x_{n}\right)+\left(1-\alpha_{n}\right) V_{\tau_{n}} x_{n}-z\right\|^{2}+M_{2}\left\|\tilde{e}_{n}\right\| \\
= & \left\|V_{\tau_{n}} x_{n}-z+\alpha_{n}\left(h_{n}\left(x_{n}\right)-V_{\tau_{n}} x_{n}\right)\right\|^{2}+M_{2}\left\|\tilde{e}_{n}\right\| \\
= & \left\|V_{\tau_{n}} x_{n}-z\right\|^{2}+\alpha_{n}{ }^{2}\left\|h_{n}\left(x_{n}\right)-V_{\tau_{n}} x_{n}\right\|^{2}+2 \alpha_{n}\left\langle V_{\tau_{n}} x_{n}-z, h_{n}\left(x_{n}\right)-V_{\tau_{n}} x_{n}\right\rangle+M_{2}\left\|\tilde{e}_{n}\right\| \\
= & \left\|\left(1-w_{n}\right) x_{n}+w_{n} T_{n} x_{n}-z\right\|^{2}+\alpha_{n}{ }^{2}\left\|h_{n}\left(x_{n}\right)-V_{\tau_{n}} x_{n}\right\|^{2} \\
& +2 \alpha_{n}\left\langle V_{\tau_{n}} x_{n}-z, h_{n}\left(x_{n}\right)-V_{\tau_{n}} x_{n}\right\rangle+M_{2}\left\|\tilde{e}_{n}\right\| \\
= & \left(1-w_{n}\right)\left\|x_{n}-z\right\|^{2}+w_{n}\left\|T_{n} x_{n}-T_{n} z\right\|^{2}-w_{n}\left(1-w_{n}\right)\left\|T_{n} x_{n}-x_{n}\right\|^{2} \\
& +\alpha_{n}{ }^{2}\left\|h_{n}\left(x_{n}\right)-V_{\tau_{n}} x_{n}\right\|^{2}+2 \alpha_{n}\left\langle V_{\tau_{n}} x_{n}-z, h_{n}\left(x_{n}\right)-V_{\tau_{n}} x_{n}\right\rangle+M_{2}\left\|\tilde{e}_{n}\right\| \\
\leq & \left\|x_{n}-z\right\|^{2}-w_{n}\left(1-w_{n}\right)\left\|T_{n} x_{n}-x_{n}\right\|^{2}+\alpha_{n}{ }^{2}\left\|h_{n}\left(x_{n}\right)-V_{\tau_{n}} x_{n}\right\|^{2} \\
& +2 \alpha_{n}\left\langle V_{\tau_{n}} x_{n}-z, h_{n}\left(x_{n}\right)-V_{\tau_{n}} x_{n}\right\rangle+M_{2}\left\|\tilde{e}_{n}\right\| .
\end{aligned}
$$

Set

$$
\begin{gathered}
s_{n}=\left\|x_{n}-z\right\|^{2}, \quad \gamma_{n}=\alpha_{n}\left(2-\alpha_{n}\left(1+2 \rho_{u}^{2}\right)-2\left(1-\alpha_{n}\right) \rho_{u}\right), \\
\delta_{n}=\frac{2\left(1-\alpha_{n}\right)\left\langle h_{n}(z)-z, V_{\tau_{n}} x_{n}-z\right\rangle+2 \alpha_{n}\left\|h_{n}(z)-z\right\|^{2}+M_{2} \frac{\left\|\tilde{e}_{n}\right\|}{\alpha_{n}}}{2-\alpha_{n}\left(1+2 \rho_{u}^{2}\right)-2\left(1-\alpha_{n}\right) \rho_{u}} \\
\eta_{n}=w_{n}\left(1-w_{n}\right)\left\|T_{n} x_{n}-x_{n}\right\|^{2}
\end{gathered}
$$

and

$$
\varphi_{n}=\alpha_{n}^{2}\left\|h_{n}\left(x_{n}\right)-V_{\tau_{n}} x_{n}\right\|^{2}+2 \alpha_{n}\left\langle V_{\tau_{n}} x_{n}-z, h_{n}\left(x_{n}\right)-V_{\tau_{n}} x_{n}\right\rangle+M_{2}\left\|\tilde{e}_{n}\right\| .
$$

Note that $\gamma_{n} \rightarrow 0, \Sigma_{n=0}^{\infty} \gamma_{n}=\infty$ and $\varphi_{n} \rightarrow 0$ as $n \rightarrow \infty$. In order to complete the proof by using Lemma 2.4, it suffices to verify that $\eta_{n_{k}} \rightarrow 0(k \rightarrow \infty)$ implies that $\limsup _{k \rightarrow \infty} \delta_{n_{k}} \leq 0$ for any subsequence $\left(n_{k}\right) \subset(n)$. Indeed, as $k \rightarrow \infty, \eta_{n_{k}} \rightarrow 0$ implies that $\left\|T_{n_{k}} x_{n_{k}}-x_{n_{k}}\right\| \rightarrow 0$ due to condition (iii). Thus, from (3.7), we have

$$
\left\|x_{n_{k}}-V_{\tau_{n_{k}}} x_{n_{k}}\right\|=w_{n_{k}}\left\|x_{n_{k}}-T_{n_{k}} x_{n_{k}}\right\| \rightarrow 0
$$

Step 3. Show that

$$
\omega_{w}\left(x_{n_{k}}\right) \subset S,
$$

where $\omega_{w}\left(x_{n_{k}}\right)$ is the set of all weak cluster points of $\left\{x_{n_{k}}\right\}$. To see (3.10), we take $\tilde{x} \in \omega_{w}\left\{x_{n_{k}}\right\}$ and assume that $\left\{x_{n_{k_{j}}}\right\}$ is a subsequence of $\left\{x_{n_{k}}\right\}$ weakly converging to $\tilde{x}$. Without loss of generality, we still use $\left\{x_{n_{k}}\right\}$ to denote $\left\{x_{n_{k_{j}}}\right\}$. Since $\tau_{n_{k}}$ is bounded, we can assume $\tau_{n_{k}} \rightarrow \tau$. Then $0<\tau<\frac{2}{L}$. Setting

$$
V_{\tau}=J_{\lambda}^{B_{1}}\left(I-\tau A^{*}\left(I-J_{\lambda}^{B_{2}}\right) A\right)
$$


we have that $V_{\tau}$ is nonexpansive. It follows that

$$
\begin{aligned}
\left\|V_{\tau_{n_{k}}} x_{n_{k}}-V_{\tau} x_{n_{k}}\right\| & =\left\|J_{\lambda}^{B_{1}}\left(x_{n_{k}}-\tau_{n_{k}} A^{*}\left(I-J_{\lambda}^{B_{2}}\right) A x_{n_{k}}\right)-J_{\lambda}^{B_{1}}\left(x_{n_{k}}-\tau A^{*}\left(I-J_{\lambda}^{B_{2}}\right) A x_{n_{k}}\right)\right\| \\
& \leq\left\|\tau_{n_{k}} A^{*}\left(I-J_{\lambda}^{B_{2}}\right) A x_{n_{k}}-\tau A^{*}\left(I-J_{\lambda}^{B_{2}}\right) A x_{n_{k}}\right\| \\
& \leq\left|\tau_{n_{k}}-\tau\right|\left\|A^{*}\left(I-J_{\lambda}^{B_{2}}\right) A x_{n_{k}}\right\| .
\end{aligned}
$$

Since $\tau_{n_{k}} \rightarrow \tau$ as $k \rightarrow \infty$, we immediately derive from the last relation that $\left\|V_{\tau_{n_{k}}} x_{n_{k}}-V_{\tau} x_{n_{k}}\right\| \rightarrow 0$. As a result, we find

$$
\left\|x_{n_{k}}-V_{\tau} x_{n_{k}}\right\| \leq\left\|x_{n_{k}}-V_{\tau_{n_{k}}} x_{n_{k}}\right\|+\left\|V_{\tau_{n_{k}}} x_{n_{k}}-V_{\tau} x_{n_{k}}\right\| \rightarrow 0
$$

Using Lemma 2.3, we get $\omega_{w}\left(x_{n_{k}}\right) \subset F i x\left(V_{\tau}\right)$. By Lemma 1.1, we obtain $\omega_{w}\left(x_{n_{k}}\right) \subset S$ immediately. Since $\left\{h_{n}(x)\right\}$ is uniformly convergent to $h(x)$ on $E$, we have $\lim _{n \rightarrow \infty}\left(h_{n}\left(x^{*}\right)-x^{*}\right)=$ $h\left(x^{*}\right)-x^{*}$. Meanwhile, we have

$$
\limsup _{k \rightarrow \infty}\left\langle h_{n_{k}}\left(x^{*}\right)-x^{*}, x_{n_{k}}-x^{*}\right\rangle=\left\langle h\left(x^{*}\right)-x^{*}, \tilde{x}-x^{*}\right\rangle, \quad \forall \tilde{x} \in S .
$$

Since $x^{*}$ is the unique solution to variational inequality problem (3.1). We get

$$
\limsup _{k \rightarrow \infty}\left\langle h_{n_{k}}\left(x^{*}\right)-x^{*}, x_{n_{k}}-x^{*}\right\rangle \leq 0
$$

and hence $\lim \sup _{k \rightarrow \infty} \delta_{n_{k}} \leq 0$. This completes the proof.

Consider the bounded perturbation of (3.4) by the following iterative form:

$$
\left\{\begin{array}{l}
y_{n}=x_{n}+\beta_{n} v_{n}, \\
x_{n+1}=\alpha_{n} h_{n}\left(y_{n}\right)+\left(1-\alpha_{n}\right) J_{\lambda}^{B_{1}}\left(\left(I-\tau_{n} A^{*}\left(I-J_{\lambda}^{B_{2}}\right) A\left(y_{n}\right)+e\left(y_{n}\right)\right),\right.
\end{array}\right.
$$

where $\tau_{n} \in\left(0, \frac{2}{L}\right)$. Similarly, we put

$$
\tilde{e}_{n}=J_{\lambda}^{B_{1}}\left(\left(I-\tau_{n} A^{*}\left(I-J_{\lambda}^{B_{2}}\right) A\left(y_{n}\right)+e\left(y_{n}\right)\right)-J_{\lambda}^{B_{1}}\left(I-\tau_{n} A^{*}\left(I-J_{\lambda}^{B_{2}}\right) A\left(y_{n}\right)\right) .\right.
$$

Theorem 3.2. Assume that the sequences $\left\{\beta_{n}\right\}$ and $\left\{v_{n}\right\}$ satisfy the condition (1.4). Let $H_{1}, H_{2}$ be two real Hilbert spaces and $A$ be a bounded linear operator with $L=\left\|A^{*} A\right\|$, where $A^{*}$ is the adjoint of $A$. Suppose that $B_{1}: H_{1} \rightarrow 2^{H_{1}}$ and $B_{2}: H_{2} \rightarrow 2^{H_{2}}$ are maximal monotone operators and $S \neq \emptyset$. Let $h_{n}$ be a $\rho_{n}$-contractive self-maps of $H_{1}$ with $0 \leq \rho_{l}=\liminf _{n \rightarrow \infty} \rho_{n} \leq$ $\limsup _{n \rightarrow \infty} \rho_{n}=\rho_{u}<1$. Suppose the contractive mapping sequence $\left\{h_{n}(x)\right\}$ is uniformly convergent for any $x \in E$, where $E$ is any bounded subset of $H_{1}$. Given $x_{0} \in H_{1}$, define the sequence $\left\{x_{n}\right\}$ by (3.14). Suppose that

(i) $\lim _{n \rightarrow \infty} \alpha_{n}=0$;

(ii) $\sum_{n=0}^{\infty} \alpha_{n}=\infty$;

(iii) $0<\liminf _{n \rightarrow \infty} \tau_{n} \leq \limsup _{n \rightarrow \infty} \tau_{n}<\frac{2}{L}$;

(iv) $\sum_{n=0}^{\infty}\left\|e\left(y_{n}\right)\right\|<\infty$.

Then $\left\{x_{n}\right\}$ converges strongly to $x^{*}$, where $x^{*}$ is a solution of the SVIP (1.1)-(1.2), which is also the unique solution of variational inequality problem (3.1).

Proof. We can rewrite (3.14) as

$$
x_{n+1}=\alpha_{n} h_{n}\left(x_{n}\right)+\left(1-\alpha_{n}\right)\left(J_{\lambda}^{B_{1}}\left(I-\tau_{n} A^{*}\left(I-J_{\lambda}^{B_{2}}\right) A\left(x_{n}\right)\right)+\tilde{e}_{n}\right)+\bar{e}_{n},
$$


where

$$
\bar{e}_{n}=\alpha_{n}\left(h_{n}\left(y_{n}\right)-h_{n}\left(x_{n}\right)\right)+\left(1-\alpha_{n}\right)\left(J_{\lambda}^{B_{1}}\left(I-\tau_{n} A^{*}\left(I-J_{\lambda}^{B_{2}}\right) A\right) y_{n}-J_{\lambda}^{B_{1}}\left(I-\tau_{n} A^{*}\left(I-J_{\lambda}^{B_{2}}\right) A\right) x_{n}\right),
$$

In fact, it is not hard to get

$$
\begin{aligned}
\left\|\bar{e}_{n}\right\| & \leq \alpha_{n}\left\|h_{n}\left(y_{n}\right)-h_{n}\left(x_{n}\right)\right\|+\left(1-\alpha_{n}\right)\left\|y_{n}-x_{n}-\tau_{n}\left(A^{*}\left(I-J_{\lambda}^{B_{2}}\right) A\left(y_{n}\right)-A^{*}\left(I-J_{\lambda}^{B_{2}}\right) A\left(x_{n}\right)\right)\right\| \\
& \leq \alpha_{n} \rho_{n} \beta_{n}\left\|v_{n}\right\|+\left(1-\alpha_{n}\right)\left(\beta_{n}\left\|v_{n}\right\|+\lambda_{n} L \| y_{n}-x_{n}\right) \| \\
& \leq\left(\alpha_{n} \rho_{u}+\left(1-\alpha_{n}\right)\left(1+\lambda_{n} L\right)\right) \beta_{n}\left\|v_{n}\right\| .
\end{aligned}
$$

From condition (1.4), we have $\sum_{n=0}^{\infty}\left\|\bar{e}_{n}\right\|<\infty$. Thus, using the Theorem 3.1, we get the algorithm (3.4) is bounded perturbation resilient.

Remark 3.1. Theorem 3.1 is a outer perturbation algorithm. We replace it by the following algorithm:

$$
x_{n+1}=\alpha_{n} h_{n}\left(x_{n}+e\left(x_{n}\right)\right)+\left(1-\alpha_{n}\right) J_{\lambda}^{B_{1}}\left(I-\tau_{n} A^{*}\left(I-J_{\lambda}^{B_{2}}\right) A\right)\left(x_{n}+e\left(x_{n}\right)\right),
$$

It is obvious that it is a inner perturbation version. Similarly, we rewrite it as

$$
x_{n+1}=\alpha_{n} h_{n}\left(x_{n}\right)+\left(1-\alpha_{n}\right) J_{\lambda}^{B_{1}}\left(I-\tau_{n} A^{*}\left(I-J_{\lambda}^{B_{2}}\right) A\right)\left(x_{n}\right)+\hat{e}_{n} .
$$

It is easy to prove

$$
\left\|\hat{e}_{n}\right\| \leq\left\|e\left(x_{n}\right)\right\|
$$

Under appropriate conditions, we can obtain strong convergence theorems.

\section{NUMERICAL RESULTS}

In this section, we consider two simple numerical examples to demonstrate the effectiveness, realization, and convergence of the Theorem 3.1.

Example 4.1. Let $H_{1}=H_{2}=\mathbb{R}^{2}$. Define $h_{n}(x)=\frac{n}{10(n+1)} x$. Take $B_{1}: \mathbb{R}^{2} \rightarrow \mathbb{R}^{2}$ and $B_{2}: \mathbb{R}^{2} \rightarrow \mathbb{R}^{2}$ as follows:

$$
B_{1}(x)=T_{1}(x) \text { and } B_{2}(x)=T_{2}(x)
$$

where

$$
T_{1}=\left(\begin{array}{ll}
8 & 0 \\
0 & 2
\end{array}\right)
$$

and

$$
T_{2}=\left(\begin{array}{ll}
3 & 0 \\
0 & 6
\end{array}\right) .
$$

Observe $T_{1}$ and $T_{2}$ are positive linear operators, and then they are maximal monotone. So, we obtain the resolvent operators $J_{\lambda}^{B_{1}}=\left(I+\lambda B_{1}\right)^{-1}$ and $J_{\lambda}^{B_{2}}=\left(I+\lambda B_{2}\right)^{-1}$, respectively. The detailed expressions are calculated as follows:

$$
J_{\lambda}^{B_{1}}=\frac{1}{(8 \lambda+1)(2 \lambda+1)}\left(\begin{array}{cc}
2 \lambda+1 & 0 \\
0 & 8 \lambda+1
\end{array}\right)
$$

and

$$
J_{\lambda}^{B_{2}}=\frac{1}{(3 \lambda+1)(6 \lambda+1)}\left(\begin{array}{cc}
6 \lambda+1 & 0 \\
0 & 3 \lambda+1
\end{array}\right) .
$$


Generate a $2 \times 2$ random matrix $A$, and compute the Lipschitz constant $L=\left\|A^{T} A\right\|$, where $A^{T}$ represents the transpose of $A$. Take $\lambda=0.5, \tau_{n}=\tau=\frac{1}{100 * L}$ and $\alpha_{n}=\frac{1}{n+1}$. According to the iterative process of Theorem 3.1, the sequence $\left\{x_{n}\right\}$ is defined by

$$
x_{n+1}=\frac{1}{n+1} * \frac{1}{10} x_{n}+\left(1-\frac{1}{n+1}\right) J_{\lambda}^{B_{1}}\left(x_{n}-\frac{1}{100 L} A^{T}\left(I-J_{\lambda}^{B_{2}}\right) A x_{n}\right) .
$$

As $n \rightarrow \infty$, we have $x_{n} \rightarrow x^{*}$. By taking random initial guess $x_{0}$, and using software MATLAB, we obtain the numerical experiment results in Table 1.

TABLE $1 . x_{0}=\operatorname{rand}(2,1)$.

\begin{tabular}{ccccc}
\hline$\tau=\frac{1}{100 * L}$ & $\mathrm{n}$ & $\operatorname{time}(s)$ & $x_{n}$ & $\operatorname{err}\left(\left\|x_{n+1}-x_{n}\right\|\right)$ \\
\hline 0.0051 & 16 & 0.000064 & $10^{-6} *[-0.00140 .9087]^{T}$ & $6.1812 * 10^{-7}$ \\
\hline 0.0143 & 23 & 0.000087 & $10^{-7} *[-0.00020 .1537]^{T}$ & $7.9865 * 10^{-9}$ \\
\hline 0.0067 & 26 & 0.000098 & $10^{-8} *[-0.00040 .1602]^{T}$ & $8.2823 * 10^{-10}$ \\
\hline
\end{tabular}

Next, we consider the algorithm with bounded perturbation resilience. Choose the bounded sequence $\left\{v_{n}\right\}$ and the summarable nonnegative real sequence $\left\{\beta_{n}\right\}$ as follows:

$$
v_{n}=\left\{\begin{aligned}
-\frac{d_{n}}{\left\|d_{n}\right\|}, & \text { if } 0 \neq d_{n} \in B_{1}\left(x_{n}\right), \\
0, & \text { if } 0 \in B_{1}\left(x_{n}\right) .
\end{aligned}\right.
$$

$\beta_{n}=c^{n}$, for some $c \in(0,1)$. Set $c=0.5$. The numerical results can be seen in Table 2 .

TABLE 2. $x_{0}=\operatorname{rand}(2,1)$.

\begin{tabular}{ccccc}
\hline$\tau=\frac{1}{100 * L}$ & $\mathrm{n}$ & time $(s)$ & $x_{n}$ & $\operatorname{err}\left(\left\|x_{n+1}-x_{n}\right\|\right)$ \\
\hline 0.0056 & 22 & 0.002426 & $10^{-6} *[0.1072-0.0003]^{T}$ & $6.9097 * 10^{-8}$ \\
\hline 0.0045 & 25 & 0.002636 & $10^{-7} *[0.10710 .0000]^{T}$ & $8.6093 * 10^{-9}$ \\
\hline 0.0046 & 29 & 0.004276 & $10^{-8} *[0.66930 .0002]^{T}$ & $5.3830 * 10^{-10}$ \\
\hline
\end{tabular}

As we have seen, the error of the solution becomes smaller as the increasing iterative numbers. And, the sequence $\left\{x_{n}\right\}$ converges $(0,0)$, which is the solution of the numerical example. Of course, it is also the unique solution of the variational inequality $\left\langle(I-h) x^{*}, x-x^{*}\right\rangle \geq 0$.

Example 4.2. Let $H_{1}=\mathbb{R}^{3}, H_{2}=\mathbb{R}^{2}$. Define $h_{n}(x)=h(x)=\frac{1}{15} x$. Take $B_{1}: \mathbb{R}^{3} \rightarrow \mathbb{R}^{3}$ and $B_{2}: \mathbb{R}^{2} \rightarrow \mathbb{R}^{2}$ as follows:

$$
B_{1}(x)=N_{C}(x) \text { and } B_{2}(x)=N_{Q}(x),
$$

where $N_{C}$ denote the normal cone to $C$ defined by

Suppose

$$
N_{C}(x)= \begin{cases}\{u \in H:\langle u, x-y\rangle \geq 0, \forall y \in C\}, & \text { if } x \in C, \\ \emptyset, & \text { if } x \notin C .\end{cases}
$$

$$
A=\left(\begin{array}{lll}
2 & 0 & 3 \\
1 & 4 & 0
\end{array}\right)
$$




$$
C=\left\{x \in \mathbb{R}^{3}:\|x\|_{2} \leq 1\right\}
$$

and

$$
Q=\left\{y \in \mathbb{R}^{2}: 1 \leq y(i) \leq 2, \quad i=1,2\right\}
$$

where $y(i)$ denotes the ith element of $y$. Given the parameters $\alpha_{n}=1 / \sqrt{n+1}, \tau_{n}=1 / L$, we obtain the following iterative form:

$$
x_{n+1}=\frac{1}{\sqrt{n+1}} * \frac{1}{10} x_{n}+\left(1-\frac{1}{\sqrt{n+1}}\right) P_{C}\left(x_{n}-\frac{1}{L} A^{T}\left(I-P_{Q}\right) A x_{n}\right) .
$$

\begin{tabular}{|c|c|c|c|c|c|}
\hline$\tau=\frac{1}{L}$ & $\mathrm{n}$ & time $(s)$ & $x_{n}$ & $y_{n}$ & eps \\
\hline 0.056 & 62 & 0.000001 & {$\left[\begin{array}{llll}0.1862 & 0.2001 & 0.2031\end{array}\right]^{T}$} & {$\left[\begin{array}{lll}0.9817 & 0.9866\end{array}\right]^{T}$} & $10^{-4}$ \\
\hline 0.056 & 597 & 0.00012 & {$\left[\begin{array}{llll}0.1886 & 0.2025 & 0.2072\end{array}\right]^{T}$} & {$\left[\begin{array}{lll}0.9988 & 0.9986\end{array}\right]^{T}$} & $10^{-6}$ \\
\hline 0.056 & 6028 & 0.2105 & {$\left[\begin{array}{llll}0.1889 & 0.2028 & 0.2074\end{array}\right]^{T}$} & {$\left[\begin{array}{lll}1.0000 & 1.0000\end{array}\right]^{T}$} & $10^{-8}$ \\
\hline
\end{tabular}

Taking random initial vector $x_{0} \in \mathbb{R}^{3}$, we get the numerical results in Table 3 .

TABLE 3. $x_{0}=\operatorname{rand}(3,1)$.

Consider the bounded perturbation of (4.3). The definitions of $v_{n}$ and $\beta_{n}$ are similar to Example 4.1. The corresponding numerical results are shown in Table 4.

TABLE 4. $x_{0}=\operatorname{rand}(3,1)$.

\begin{tabular}{cccccc}
\hline$\tau=\frac{1}{L}$ & $\mathrm{n}$ & time $(s)$ & $x_{n}$ & $y_{n}$ & eps \\
\hline 0.056 & 62 & 0.000001 & {$\left[\begin{array}{lllll}0.1859 & 0.2001 & 0.2031\end{array}\right]^{T}$} & {$\left[\begin{array}{llll}0.9811 & 0.9862\end{array}\right]^{T}$} & $10^{-4}$ \\
\hline 0.056 & 568 & 0.0001 & {$\left[\begin{array}{lllll}0.1886 & 0.2025 & 0.2070\end{array}\right]^{T}$} & {$\left[\begin{array}{llll}0.9981 & 0.9986\end{array}\right]^{T}$} & $10^{-6}$ \\
\hline 0.056 & 6005 & 0.1980 & {$\left[\begin{array}{lllll}0.1889 & 0.2028 & 0.2074\end{array}\right]^{T}$} & {$\left[\begin{array}{llll}1.0000 & 1.0000\end{array}\right]^{T}$} & $10^{-8}$ \\
\hline
\end{tabular}

From the point of view of calculation, the above algorithms in the examples are easy to implement.

\section{Acknowledgments}

The authors would like to thank the handling editors and the anonymous referees for valuable suggestions which improved the manuscript. This paper was supported by the Scientific Research Project of Tianjin Municipal Education Commission(2018KJ253) and the Foundation of Tianjin Key Laboratory for Advanced Signal Processing.

\section{REFERENCES}

[1] H.H. Bauschke, P.L. Combettes, Convex Analysis and Monotone Operator Theory in Hilbert Spaces, Springer, New York, 2011.

[2] J.Y. Bello Cruz, Y. Shehu, An iterative method for split inclusion problems without prior knowledge of operator norms, J. Fixed Point Theory Appl. 19 (2017), 2017-2036. 
[3] D. Butnariu, R. Davidi, G.T. Herman, I.G. Kazantsev, Stable convergence behavior under summable perturbation of a class projection method for convex feasibility and optimization problems, IEEE J. Selected Topics Signal Process, 1 (2007), 540-547.

[4] C. Byrne, Iterative oblique projection onto convex sets and the split feasibility problem, Inverse probl. 18 (2002), 441-453.

[5] C. Byrne, Y. Censor, A. Gibali, S. Reich, Weak and strong convergence of algorithms for the split common null point problem, J. Nonlinear convex Anal. 13 (2012), 759-775.

[6] P.L. Combettes, Solving monotone inclusions via compositions of nonexpansive averaged operators, Opitimization, 53 (2004), 475-504.

[7] Y. Censor, T. Elfving, A multiprojection algorithm using Bregman projections in product space, Numer. Algo. 8 (1994), 221-239.

[8] Y. Censor, T. Bortfeld, B. Martin, A. Trofimov, A unified approach for inversion problems in intensity modulated radiation therapy, Phys. Med. Biol. 51 (2006), 2353-2365.

[9] Y. Censor, R. Davidi, G.T. Herman, Perturbation resilience and superiorization of iterative algorithms. Inverse Probl. 26 (2010), 065008.

[10] Y. Censor, W. Chen, P.L. Combettes, R. Davidi, G.T. Herman, On the effectiveness of projection methods for convex feasibility problem with linear inequality constrains, Comput. Optim. Appl. 51 (2012), 1065-1088.

[11] H.T. Che, M.X. Li, The conjugate gradient method for split variational inclusion and constrained convex minimization problems, Appl. Math. Comput. 290 (2016), 426-438.

[12] C.S. Chuang, Strong convergence theorems for the split variational inclusion problem in Hilbert spaces, Fixed Point Theory Appl. 2013 (2013), 350.

[13] C.S. Chuang, L.J. Lin, New strong convergence theorems for split variational inclusion problems in Hilbert spaces, J. Inequal. Appl. 76 (2015), 176.

[14] R. Davidi, Y. Censor, R.W. Schulte, S. Geneser, L. Xing, Feasibility-seeking and superiorization algorithm applied to inverse treatment plannning in rediation therapy. Contemp. Math. 636 (2015), 83-92.

[15] R. Davidi, G.T. Herman, Y. Censor, Perturbation-resilient block-iterative projection methods with application to image reconstruction from projections, Int. Trans. Oper. Res. 16 (2009), 505-524.

[16] Q.L. Dong, J. Zhao, S.N. He, Bounded perturbation resilience of the viscosity algorithm, J. Inequal. Appl. 2016 (2016), 299.

[17] K. Geobel, W.A. Kirk, Topics in metric fixed point theory, Cambridge Studies in Advanced Mathematics, Cambridge University Press, Cambridge, 1990.

[18] Y. Guo, W. Cui, Strong convergence and bounded perturbation resilience of a modified proximal gradient algorithm, J. Inequal. Appl. 2018 (2018), 103.

[19] S.N. He, C.P. Yang, Solving the variational inequality problem defined on intersection of finite level sets, Abstr. Appl. Anal. 2013 (2013), 942315.

[20] W. Jin, Y. Censor, M. Jiang, Bounded perturbation resilience of projected scaled gradient methods, Comput. Optim. Appl. 63 (2016), 365-392.

[21] K. Kazmi, S. Rizvi, An iterative method for split variational inclusion problem and fixed point problem for a nonexpansive mapping, Optim. Lett. 8 (2014), 1113-1124.

[22] A. Moudafi, Viscosity approximation methods for fixed point problems, J. Math. Anal. Appl. 241 (2000), 46-55.

[23] A. Moudafi, Split monotone variational inclusions, J. Optim. Theory Appl. 150 (2011), 275-283.

[24] X. Qin, S.Y. Cho, L. Wang, Iterative algorithms with errors for zero points of $\mathrm{m}$-accretive operators, Fixed Point Theory Appl. 2013 (2013), 148.

[25] X. Qin, S.Y. Cho, J.C. Yao, A viscosity iterative method for a split feasibility problem, J. Nonlinear Convex Anal. 20 (2019), 1497-1506.

[26] W. Takahashi, H.K. Xu, J.C. Yao, Iterative methods for generalized split feasibility problems in Hilbert space, Set-Valued Var. Anal. 23 (2015), 205-221.

[27] H.K. Xu, Averaged mappings and the gradient-projection algorithm, J. Optim. Theory Appl. 150 (2011), 360-378.

[28] H.K. Xu, Viscosity approximation methods for nonexpansive mappings, J. Math. Anal. Appl. 298 (2004), 279-291. 
[29] H.K. Xu, Bounded perturbation resilience and superiorization techniques for the projected scaled gradient method, Inverse Probl. 33 (2017), 044008.

[30] C.Y. Yang, S.N. He, General alterative regularization methods for nonexpansive mappings in Hilbert spaces, Fixed Point Theory Appl. 2014 (2014), 203. 